\title{
THE INFLUENCE OF LIMITED DYNAMIC RESPONSE OF THE INDICATOR DETECTOR IN A SWAN-GANZ CATHETER ON THE OVERESTIMATION OF CARDIAC OUTPUT MEASUREMENT BY MEANS OF THERMODILUTION
}

\author{
Maciej Gawlikowski' ${ }^{1)}$, Tadeusz Pustelny ${ }^{2)}$ \\ 1) Foundation of Cardiac Surgery Development, Wolności 345a, 41-800 Zabrze, Poland \\ $(\bowtie$ maciej.gawlikowski@poczta.onet.pl, +48 32373 5663) \\ 2) Silesian University of Technology, Faculty of Electrical Engineering, Krzywoustego 2, 44-101 Gliwice, Poland \\ (tadeusz.pustelny@polsl.pl)
}

\begin{abstract}
Nowadays the "gold clinical standard" of hemodynamics diagnostic and cardiac output measurements is pulmonary artery catheterization by means of the Swan-Ganz catheter and thermodilution. The method itself is sensitive to numerous disturbances which cause inaccurate results. One of the well-known disadvantages of thermodilution is the overestimation of results at low values of cardiac output. This effect may concern the limited slew rate of the thermoelement mounted at the tip of the catheter. In this paper the relationship between the dynamic response of the thermoelement and the uncertainty of cardiac output measurements by means of thermodilution has been investigated theoretically and experimentally.
\end{abstract}

Keywords: cardiac output measurement, thermodilution method, Swan-Ganz catheter.

(C) 2012 Polish Academy of Sciences. All rights reserved

\section{Introduction}

Apart from intracardiac pressures, the cardiac output $(C O)$ constitutes one of the most important hemodynamics parameters $[1,18]$. In spite of numerous non-invasive methods of $C O$ measurements (e.g. blood flow generated by ventricular assist devices [2-3] or imagebased methods [17]) in the course of the last 40 years, thermodilution has achieved considerable clinical significance $[1,4]$. The examination runs as follows: first a Swan-Ganz catheter is placed into a vessel of the pulmonary circulation (this procedure is called pulmonary artery catheterization $-P A C$ ). Next, the indicator (most often $10 \mathrm{ml}$ of iced or room-temperature $0.9 \% \mathrm{NaCl}$ ) is injected through the catheter into the right atrium. The indicator is diluted by flowing blood and transferred through the right ventricle and pulmonary trunk to a detector located at one of the pulmonary arteries. A miniaturized thermoelement is applied as the detector in the Swan-Ganz catheter. The detector is connected to the patient's monitor, which measures and registers the temperature of blood $v s$. time characteristics, called the indicator dilution curve (IDC - see Fig. 1). The $C O$ is calculated based on the Stewart-Hamilton (1) and the registered IDC.

$$
Q=\frac{c_{i} \cdot \sigma_{i}}{c_{b} \cdot \sigma_{b}} \cdot \frac{V_{i} \cdot\left(T_{b 0}-T_{i}\right)}{\int_{0}^{\infty} T_{b}(t) d t},
$$

where: $c_{b}, c_{i}$ - specific heat of blood and the indicator, $\rho_{b}, \rho_{i}$ - mass density of blood and the indicator, $V_{i}$ - volume of the indicator, $T_{b 0}, T_{i}$ - temperature of blood and the indicator, $T_{b}(t)-$ indicator dilution curve. 
11
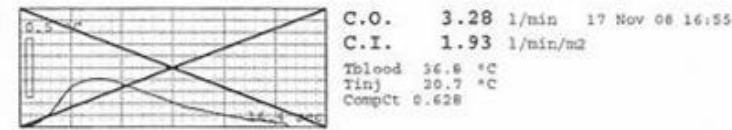

Thiood 36,6 : 1 .
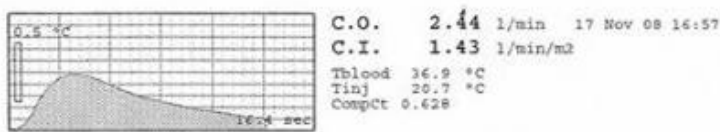

C.I. $1.43 \mathrm{inin} / \mathrm{mol}$

Thlood $36.9: C$

\#5

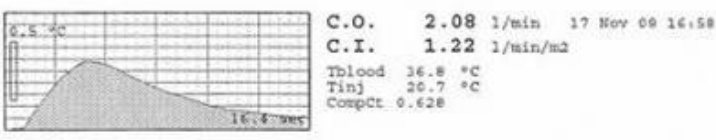

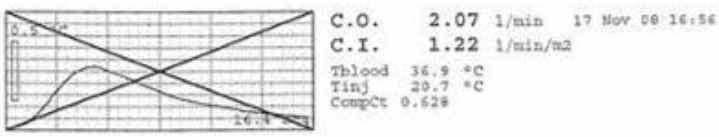

$\# 4$

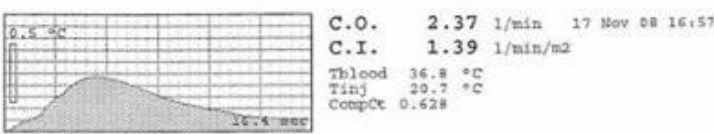

Fig. 1. The hemodynamics report of $C O$ measurements by means of thermodilution in a patient with cardiac insufficiency - demonstration of the curves of the indicator dilution $(I D C)$.

The results of $C O$ measurements by means of thermodilution are sensitive to numerous disturbances with a different physical nature. It has been pointed out in many papers [1, 5-7] that the most significant reasons of the uncertainty of thermodilution are: inaccuracy of measurements of the indicator and blood temperature, instability of the volume of the indicator, variable thermodynamic parameters of blood (e.g. caused by intravenous injection of medicines), diseases of the valves, intracardiac leaks of blood, spontaneous ventilation, arrhythmia, displacement of the catheter during the examination and thermal instability of the patient (e.g. hypothermia or an inflammatory state [8]). In spite of invasiveness and the problems mentioned above, $P A C$ and $C O$ measurements by means of thermodilution provide many valuable hemodynamics data and constitute the "gold standard" of hemodynamic diagnostics $[1,18]$.

A clinically well-known disadvantage of thermodilution is the overestimation of the results of examinations in the case of low values of $C O$ [9-11]. The meaning of this problem is particularly important in the case of patients with serious heart failures connected with cardiac contractibility impairment and decrease of ejection fraction and cardiac output. Overestimation of $C O$ may in those patients leads to incorrect diagnosis and, in consequence, contributes to taking a wrong decision concerning further therapy.

Up till now the cause of the problem mentioned above has not been unambiguously explained. One possible reason seems to be limited dynamic response of the detector of the indicator concentration, namely the thermoelement mounted at the tip of the Swan-Ganz catheter.

\section{Goal}

The goal of investigations was to assess the influence of the dynamic response of the detector on results of $\mathrm{CO}$ measurements by means of thermodilution and the Swan-Ganz catheter.

\section{Material and methods}

The following method of investigation has been assumed: the dilution process was mathematically modeled in order to determine the course of IDC. Next, by means of numerical differentiation the maximum value of the $I D C$ slew rate $(d T / d t)$ was calculated for different flows. Those data were compared with $d T / d t$ measured experimentally for the thermoelement in the Swan-Ganz catheter. 
Theoretical determination of the course of ICD was carried out by means of a mathematical, one-dimensional Local Density Random Walk model of dilution $[12,14]$. The model allows to determine the way of indicator dilution inside a rectilinear vessel containing the flowing liquid. Numerous initial assumptions of this model are, among others: impulse injection of the indicator, constant velocity of flow, the Brownian movement of the molecules of the indicator and their ideal elastic collisions with the molecules of the liquid and the lack of dissipation of the indicator through the walls of the vessel. The mathematical definition of the $L D R W$ model will be explained below.

The time dependence of the concentration of the indicator at the measurement point is defined by (2):

$$
c(t)=\frac{m_{i}}{Q} \sqrt{\frac{\lambda}{2 \Pi \cdot \mu \cdot t}} \cdot e^{\lambda-\frac{\lambda}{2}\left(\frac{t}{\mu}+\frac{\mu}{t}\right)},
$$

where: $m_{i}$ - mass of the indicator, $Q$ - volumetric flow, $\mu$ - mean transit time of the indicator between the point of injection and detection, $\lambda$ - nondimensional skewness coefficient.

The mean transit time of the indicator is calculated by means of (3):

$$
\mu=\frac{x}{Q} \cdot A,
$$

where: $x$ - distance between points of injection and measurement, $A$ - the cross-section area of the vessel.

The skewness coefficient $\lambda$ (4) concerns the Peclet number, which defines the ratio of the thermal diffusive flow to the convective flow:

$$
\lambda=\frac{\mu \cdot Q^{2}}{2 \cdot D \cdot A^{2}}
$$

where: $D$ - the diffusion coefficient $\left[\mathrm{m}^{2} / \mathrm{s}\right]$.

The diffusion coefficient (5) determines the ratio of the coefficient of thermal conductivity $[\mathrm{J} /(\mathrm{s} * \mathrm{~m} * \mathrm{~K})]$ to the volumetric thermal capacity $\left[\mathrm{J} /\left(\mathrm{m}^{3} * \mathrm{~K}\right)\right]$. Usually the diffusion coefficient has to be determined experimentally.

$$
D=\frac{R_{T}}{C_{V}}=\frac{R_{T}}{c_{w} \cdot \rho} .
$$

Numerical simulations of the course of IDC were carried out for flow in the range of $1.0-$ $5.01 / \mathrm{min}$ and for two areas of the cross-section of the vessel: $3 \mathrm{~cm}^{2}$ and $4 \mathrm{~cm}^{2}$. The maximum value of the $I D C$ slew rate was calculated by means of numerical differentiation.

Experimental investigations were carried out on the Swan-Ganz catheter (size 7F, BectonDickinson). The thermal excitation in the form of a negative temperature step was generated by a rapid plunge of the catheter into the liquid with a stabilized temperature, lower than the ambient temperature. The thermoelement mounted in the catheter was connected to a Wheatstone bridge and amplifier (Fig. 2) similar to the device applied in the patient's monitor (FX2000, Emtel). The registration of voltage (corresponding to temperature) was carried out by means of a multifunction data acquisition device with a sampling frequency of $1 \mathrm{kHz}$ (NIUSB-6221, National Instruments). Both temperatures, ambient and liquid, were measured by means of a precise electronic thermometer (Fluke 1532 and PRT probe, accuracy $0.0011^{\circ} \mathrm{C}$ ).

It was assumed that the voltage $v s$. the temperature transfer function is linear. This assumption is correct because at low increases of temperature the polynomial transmittance of the thermocouple may be approximated by a linear function with a low uncertainty. The 
temperature $v s$. voltage scaling was carried out by appropriate measurements at two different temperatures $\left(36.0^{\circ} \mathrm{C}\right.$ and $\left.37.0^{\circ} \mathrm{C}\right)$.

For all thermal excitations $N=12$ measurements were carried out. Due to the unknown variance of statistical population and low sample size, Student's distribution $(p=0.05)$ was applied to the determination of confidence limits.

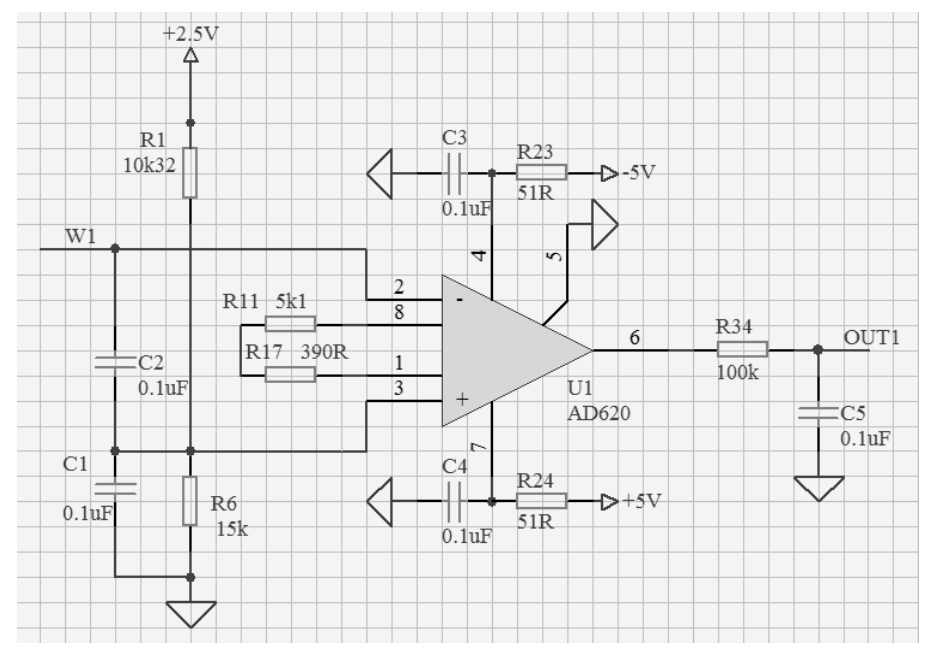

Fig. 2. Scheme of the measurement system

\section{Results}

Numerical simulations of the course of $I D C$ were carried out for flows in the range of $1.0 \ldots .0 .01 / \mathrm{min}$ and for areas of the cross-section of the vessel amounting to $3.0 \mathrm{~cm}^{2}$ and $4.0 \mathrm{~cm}^{2}$. The value of the diffusion coefficient $D=0.0500 \pm 0.0035$ was estimated experimentally by analyzing the $I D C$ curves collected during examinations of patients [12]. The demonstration courses of $I D C$ and its derivative have been presented in Fig. 3 and Fig. 4, respectively. The dependence of $d T / d t v s$. flow is to be seen in Fig. 5 .

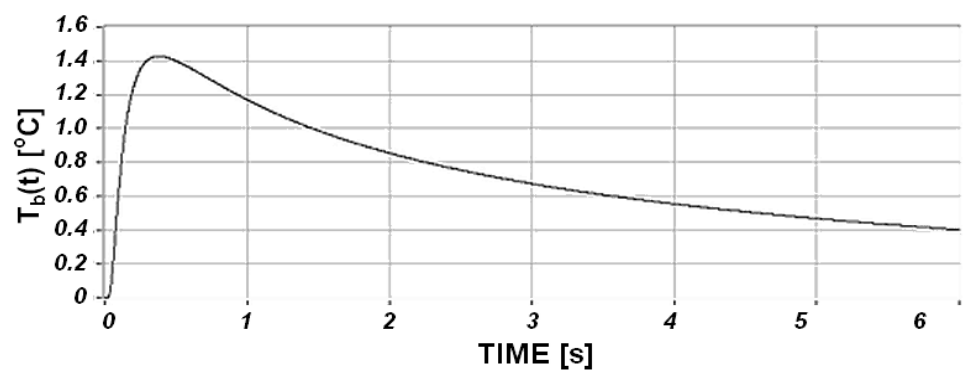

Fig. 3. The course of $I D C$ (numerical simulation).

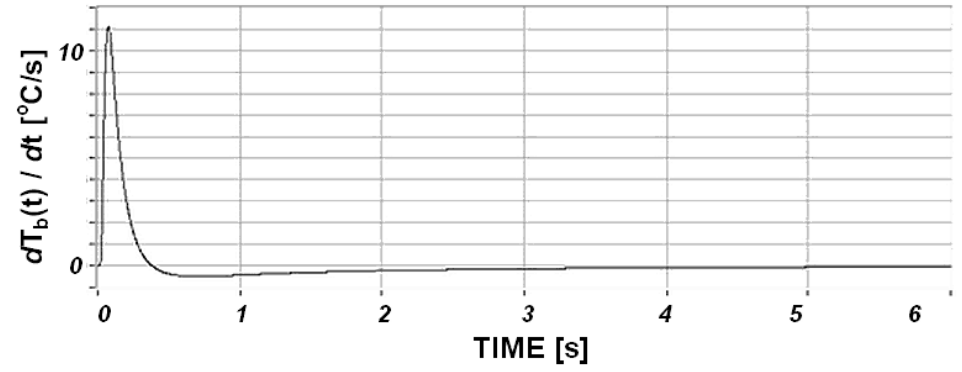

Fig. 4. The course of the derivative of $I D C$. 


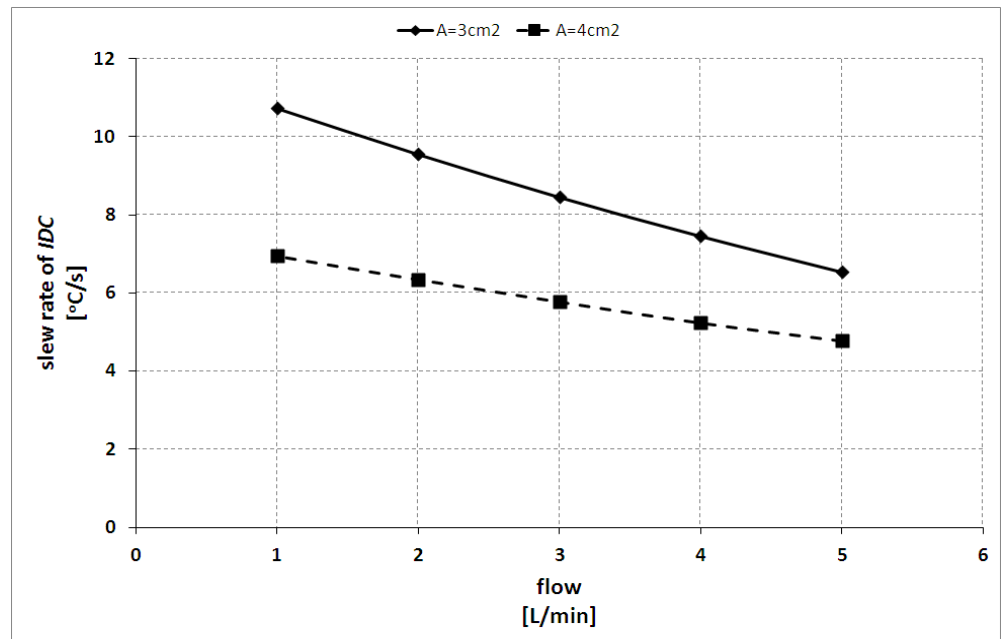

Fig. 5. Relationship between the maximum value of the $I D C$ slew rate, the flow and cross-section area of the vessel.

Physical measurements of $d T / d t$ of a thermoelement in the Swan-Ganz catheter were carried out for large values of negative thermal step excitations (Fig. 6 - solid line). The values of $d T / d t$ at low thermal excitations were assessed by means of the regression method (Fig. 6 - dashed line).

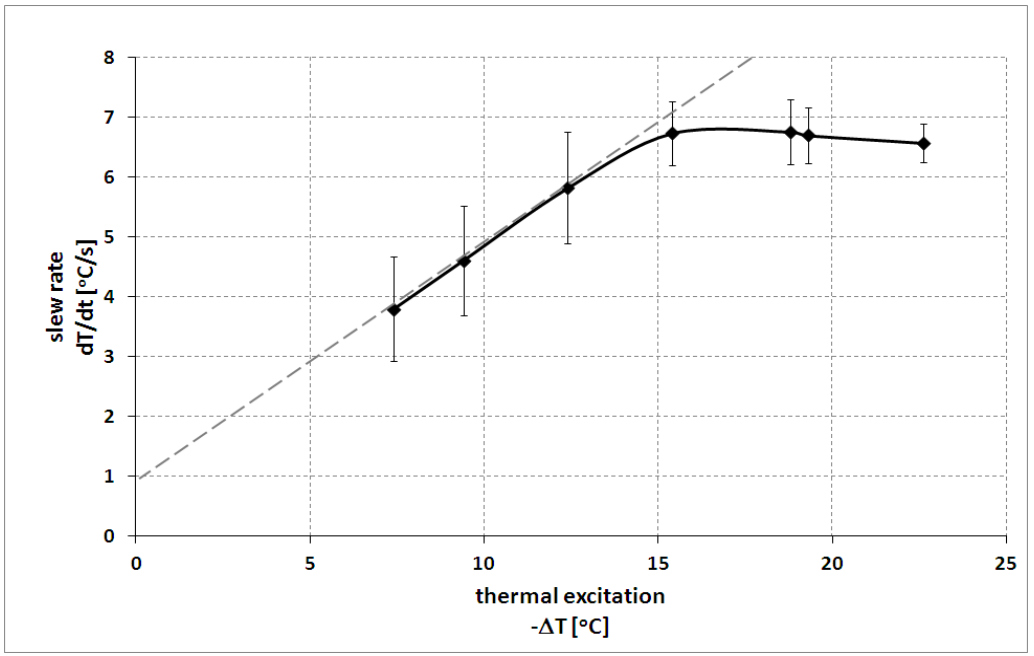

Fig. 6. The dependence of slew rate of thermoelement vs. amplitude of thermal step excitation.

For $-\Delta T<15^{\circ} \mathrm{C}$ the characteristic presented in Fig. 6 is linear and its slew rate amounts to $0.4 \mathrm{~s}^{-1}$. The $d T / d t$ estimated for low amplitudes of thermal excitation by means of linear regression amounted to $1.0^{\circ} \mathrm{C} / \mathrm{s}$.

\section{Discussion}

There are very few papers concerning the dynamic properties of the thermoelement in the Swan-Ganz catheter. One of them claims that the time constant of the thermoelement amounts to $2 \mathrm{~s}$, however the authors did not inform about the conditions of the experiments, particularly about the value of thermal excitation [15]. Assuming the exponential model of phenomena quoted by (6), the maximum value of $d T / d t$ calculated by differentiation of (6) at $t=0$ amounts to $1.25^{\circ} \mathrm{C} / \mathrm{s}$ (in the case of a typical temperature drop for iced thermodilution of 
about $2.5^{\circ} \mathrm{C}$ ). Physical measurements revealed that the $d T / d t$ value for the investigated SwanGanz catheter and a similar temperature drop amounts to $2.0^{\circ} \mathrm{C} / \mathrm{s}$.

$$
\begin{gathered}
T(t)=\left(T_{b}-T_{i}\right) \cdot e^{-\frac{t}{\tau}}-T_{i}, \\
\left.\frac{d T(t)}{d t}\right|_{t=0}=-\frac{T_{b}-T_{i}}{\tau} .
\end{gathered}
$$

Mathematical modeling of the course of ICD showed (Fig. 5) that $d T / d t$ depends on the flow and area of the cross-section of the vessel with the following relationship: the lower the flow, the greater $d T / d t$. This effect was confirmed experimentally in measurements of $C O$ by means of thermodilution carried out on the physical model of pulmonary circulation $[12,16]$. The courses of $I D C$ obtained for three values of flow $(0.98,1.81$ and $2.401 / \mathrm{min})$ are presented in Fig. 7.

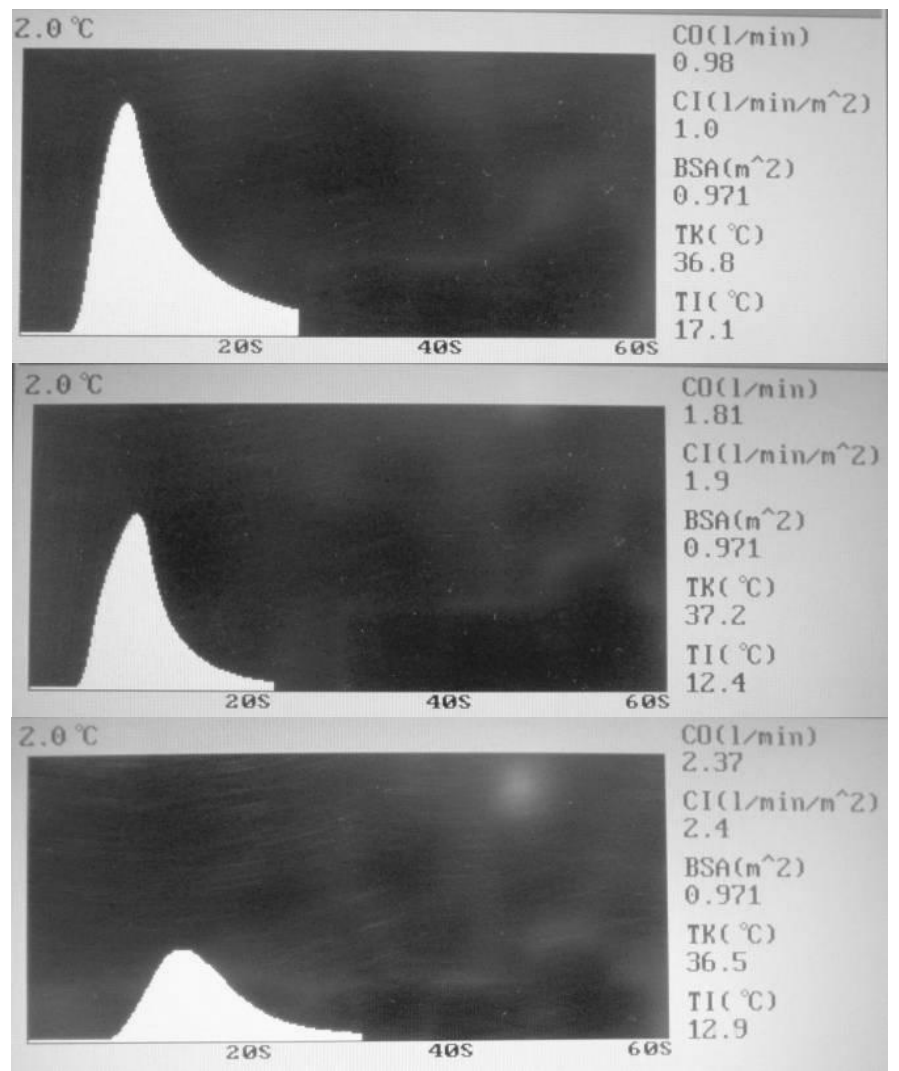

Fig. 7. Screenshots of the patient's monitor $-C O$ measurements by be means of thermodilution carried out on physical model of the pulmonary circulation.

Let us suppose that the area of the cross-section of the vessel amounts to $3 \mathrm{~cm}^{2}$ and that the blood temperature drop after injection of the indicator amounts to $2.5^{\circ} \mathrm{C}$ (iced thermodilution). Under those conditions the theoretical $d T / d t$ of $I D C$ is in the range from $10.5^{\circ} \mathrm{C} / \mathrm{s}$ to $6.5^{\circ} \mathrm{C} / \mathrm{s}$ (for flows from 1.01/min to 5.01/min, respectively, see Fig. 6), but the dynamic response of the thermoelement in the Swan-Ganz catheter is significantly lower and amounts to $2.0^{\circ} \mathrm{C} / \mathrm{s}$ (Fig. 5). This limitation causes a dynamic distortion of the IDC course which affects the value of the IDC integral and, in consequence, an uncertainty of $C O$ measurements by means of thermodilution at low flows. The mechanics of this effect have been explained in Fig. 8. At a low $C O$ the value of the slew rate in the initial part of $I D C$ is much higher than the dynamic response of the thermoelement (e.g.: the performed investigations revealed that when 
$C O=1.01 / \mathrm{min}$, the theoretical $d T / d t$ amounts to about $10^{\circ} \mathrm{C} / \mathrm{s}$ and the slew rate of the thermocouple was $2.5^{\circ} \mathrm{C} / \mathrm{s}$ ). Due to the slower dynamic response of the thermoelement than the slew rate of $I D C$, the integral of the measured $I D C$ is lower than the theoretical one (the difference has been marked in Fig. 8 in grey). Indeed, the lower the $C O$, the higher the underestimation of the integral of $I D C$. Because the integral of $I D C$ is in the denominator of Stewart-Hamilton's equation (1), the decrease of this quantity leads to an overestimation of $\mathrm{CO}$.

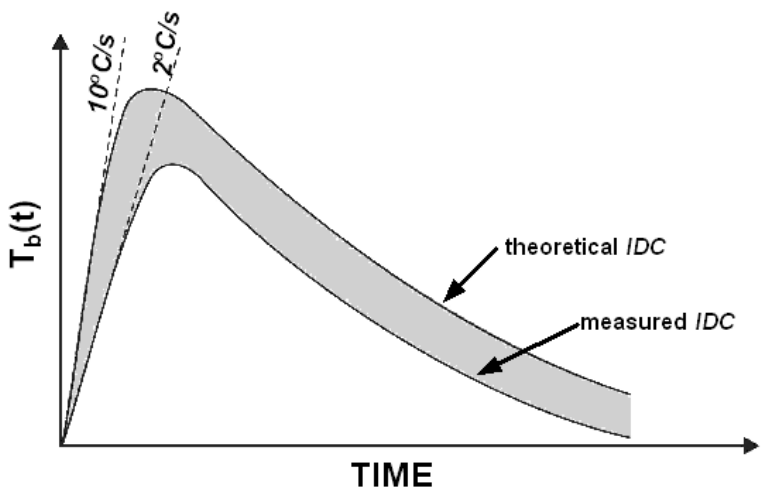

Fig. 8. Illustration of the influence of the slew rate of the temperature detector on the course and integral of $I D C$.

It should be noticed that even at a large diameter of the vessel and the value of $C O$, the slew rate of the thermodetector in the Swan-Ganz catheter is too low to measure IDC with appropriate dynamics. The effect of the dynamic response of the detector discussed in this paper influences significantly the uncertainty of all dilution methods, independently of the type of the indicator, and it seemed to be impossible to be compensated mathematically.

\section{Conclusions}

Basing on the performed investigations it may be said that one of the reasons of overestimation of the low cardiac output measured by means of thermodilution is the limited dynamic response of the thermoelement in the Swan-Ganz catheter.

\section{Acknowledgements}

This work was supported by the Polish Ministry of Science (grant No. N N518 336135) and the Czeslaw M. Rodkiewicz Scholarship Foundation.

\section{References}

[1] Headley, J.M. (2002). Invasive hemodynamic monitoring: physiological principles and clinical applications. Edwards Lifescience, Irvine.

[2] Opilski, Z., Konieczny, G., Pustelny, T., Gacek, A., Kustosz, R., Gawlikowski, M. (2011). Noninvasive acoustic blood volume measurement system for the POLVAD prosthesis. Bull. Pol. Acad. Sci.- Tech. Sci., 59(4), 429-433.

[3] Konieczny, G., Opilski, Z., Pustelny, T. (2011). Preliminary research concerning measurements of the POLVAD blood chamber volume based on Helmholtz's acoustic resonator principle. Acta Physica Polonica A, 120(6), 688-692.

[4] Mathews, L., Singh, K. (2008). Cardiac output monitoring. Annals of Cardiac Anaesthesia, 11(1), 56-68.

[5] Nishikawa, T., Doshi, S. (1993). Errors in the measurement of cardiac output by thermodilution. Canadian Journal of Anesthesia, 40(2), 142-153. 
[6] Gawlikowski, M., Pustelny, T., Przywara-Chowaniec, B., Nowak-Gawlikowska, J. (2011). Theoretical and model analysis of the unreliability of cardiac output measurement by means of the thermodilution method. Bull. Pol. Acad. Sci.- Tech. Sci., 59(4), 435-439.

[7] Gawlikowski, M., Pustelny, T. (2012). Investigations concerning the application of the cross-correlation method in cardiac output measurements. BioMedical Engineering OnLine, 11(24).

[8] Gawlikowski, M., Pustelny, T., Przywara-Chowaniec, B., Nowak-Gawlikowska, J. (2010). Model study of cardiac output measurement by thermodilution in thermal instability. Acta Physica Polonica A, 118(6), 1124-1126.

[9] Tournade, J.P., Chassard, D., Muchada, R. (1997). Overestimation of low cardiac output measured by thermodilution. British Journal of Anaesthesia, 79(4), 514-516.

[10] A.van Grondelle, R. V. Ditchey, B. M. Groves, et al.: "Thermodilution method overestimates low cardiac output in humans". American Journal of Physiology, 245(4), 1983, pp. H690-692.

[11] Maruschak, G.F., Potter, A.M., Schauble, J.F., Rogers, M.C. (1982). Overestimation of pediatric cardiac output by thermal indicator loss. Circulation, 65(2), 380-383.

[12] Sheppard, C.W., Savage, L.J. (1951). The random walk problem in relation to the physiology of circulatory mixing. Physical Review, 83, 489-490.

[13] Wise, M.E. (1966). Tracer dilution curves in cardiology and random walk and lognormal distributions. Acta Physiologica et Pharmacologica Neerlandica, 14(2), 175-204.

[14] Gawlikowski, M. (2011). Model investigation on selected metrological parameters of circulatory system for hemodynamics diagnostics. Ph.d. Dissertation. Bialystok University of Technology, Bialystok, Poland. (in Polish)

[15] dos Santos, I. (2003). In vivo measurements of the heat convection coefficient on the endocardial surface. Phd Dissertation. The University of Texas, Austin, USA.

[16] Gawlikowski, M., Pustelny, T. (2011). Physical model of the pulmonary circulation designed for investigation on cardiac output measurement by means of the thermodilution method. Acta Physica Polonica A, 120(4), 798-802.

[17] Skalski, A., Turcza, P. (2011). Heart segmentation in echo images. Metrol. Meas. Syst., 18(2), 305-314.

[18] Wtorek, J., Bujnowski, A., Rumiński, J., Poliński, A., Kaczmarek, M., Nowakowski, A. (2012). Assesment of cardiovascular risk in assisted living. Metrol. Meas. Syst., 19(2), 231-244. 